\title{
Plasma concentrations of inflammatory cytokines rise rapidly during ECMO-related SIRS due to the release of preformed stores in the intestine
}

\author{
R Britt MclLwain ${ }^{1,2}$, Joseph G Timpa ${ }^{1}$, Ashish R Kurundkarr ${ }^{3}$, David W Holt ${ }^{2}$, David R Kelly ${ }^{4}$, Yolanda E Hartman ${ }^{3}$, \\ Mary Lauren $\mathrm{Neel}^{3}$, Rajendra K Karnatak ${ }^{3}$, Robert L Schelonka ${ }^{5}, \mathrm{GM}$ Anantharamaiah ${ }^{6}$, Cheryl R Killingsworth ${ }^{6}$ \\ and Akhil Maheshwari ${ }^{3,4,7}$
}

Extracorporeal membrane oxygenation (ECMO) is a life-saving support system used in neonates and young children with severe cardiorespiratory failure. Although ECMO has reduced mortality in these critically ill patients, almost all patients treated with ECMO develop a systemic inflammatory response syndrome (SIRS) characterized by a 'cytokine storm', leukocyte activation, and multisystem organ dysfunction. We used a neonatal porcine model of ECMO to investigate whether rising plasma concentrations of inflammatory cytokines during ECMO reflect de novo synthesis of these mediators in inflamed tissues, and therefore, can be used to assess the severity of ECMO-related SIRS. Previously healthy piglets (3-week-old) were subjected to venoarterial ECMO for up to $8 \mathrm{~h}$. SIRS was assessed by histopathological analysis, measurement of neutrophil activation (flow cytometry), plasma cytokine concentrations (enzyme immunoassays), and tissue expression of inflammatory genes (PCR/western blots). Mast cell degranulation was investigated by measurement of plasma tryptase activity. Porcine neonatal ECMO was associated with systemic inflammatory changes similar to those seen in human neonates. Tumor necrosis factor-alpha (TNF- $\alpha$ ) and interleukin-8 (IL-8) concentrations rose rapidly during the first $2 \mathrm{~h}$ of ECMO, faster than the tissue expression of these cytokines. ECMO was associated with increased plasma mast cell tryptase activity, indicating that increased plasma concentrations of inflammatory cytokines during ECMO may result from mast cell degranulation and associated release of preformed cytokines stored in mast cells. TNF- $\alpha$ and IL-8 concentrations rose faster in plasma than in the peripheral tissues during ECMO, indicating that rising plasma levels of these cytokines immediately after the initiation of ECMO may not reflect increasing tissue synthesis of these cytokines. Mobilization of preformed cellular stores of inflammatory cytokines such as in mucosal mast cells may have an important pathophysiological role in ECMO-related SIRS.

Laboratory Investigation (2010) 90, 128-139; doi:10.1038/labinvest.2009.119; published online 9 November 2009

KEYWORDS: venoarterial; neonate; mast cells; intestine; CRP; cytokines

Extracorporeal membrane oxygenation (ECMO) is used for providing cardiopulmonary support in critically ill neonates and young children with respiratory failure, congenital heart disease, and overwhelming sepsis. Although the availability of ECMO has reduced mortality in these patients by nearly $80 \%$, concerns remain about the near-universal occurrence of a systemic inflammatory response syndrome (SIRS) during ECMO that is associated with multisystem organ dysfunction and considerable morbidity. This inflammatory response usually manifests within the first few hours of ECMO with hypotension, decreased urine output, decreased lung compliance, anasarca, and liver dysfunction, changes that frequently persist for several days and delay recovery from the underlying disease that led to ECMO in the first place. ${ }^{1-3}$ Elucidation of the mechanisms of ECMO-related SIRS is a critical step in the development of effective anti-in-

\footnotetext{
'University Hospital Services, University of Alabama at Birmingham (UAB), Birmingham, AL, USA; ${ }^{2}$ Clinical Perfusion Education, School of Allied Health Professions, University of Nebraska Medical Center, Omaha, NE, USA; ${ }^{3}$ Department of Pediatrics, University of Alabama at Birmingham, Birmingham, AL, USA; ${ }^{4}$ Department of Pathology, University of Alabama at Birmingham, Birmingham, AL, USA; ${ }^{5}$ Department of Pediatrics, Oregon Health and Science University, Portland, OR, USA; ${ }^{6}$ Department of Medicine, University of Alabama at Birmingham, Birmingham, AL, USA and ${ }^{7}$ Department of Cell Biology, University of Alabama at Birmingham, Birmingham, AL, USA

Correspondence: Dr A Maheshwari, MD, Department of Pediatrics, University of Alabama, 1670 University Boulevard, VH648C, Birmingham, AL 35294, USA. E-mail: akhil@peds.uab.edu

Received 4 September 2009; revised 30 September 2009; accepted 1 October 2009
} 
flammatory strategies, which may prevent adverse effects and thereby reduce the average duration of ECMO, encourage its institution earlier in the clinical course of cardiorespiratory failure rather than as a 'rescue' therapy of last resort, and possibly allow its application in a wider range of indications. ${ }^{1,3}$

Extracorporeal membrane oxygenation-related inflammatory complications are more frequent in younger patients. Neonates and young infants are at greater risk because the enormous disparity between the patient's circulating blood volume and that of the circuit (circuit volumes are often $200-300 \%$ greater than patient's circulating blood volume) increases the activation of various blood components on exposure to the foreign surface of the ECMO circuit. ${ }^{4,5}$ The high incidence of ECMO-related complications in neonates is not altered by the configuration (venoarterial $v s$ venovenous) of ECMO or by the underlying cause of cardiorespiratory failure; ${ }^{6}$ developmental factors such as high metabolic demands, reactivity of the pulmonary vasculature, and immaturity of vascular autoregulation are believed to be more important. ${ }^{5}$ Our understanding of the inflammatory effects of extracorporeal circulation is primarily based on studies on adult patients treated with cardiopulmonary bypass (CPB) during cardiac surgery. ${ }^{78}$ However, the extrapolation of data from CPB to ECMO is difficult because of major pathophysiological differences between the two modalities, such as the presence of defined ischemia-reperfusion sequences in $\mathrm{CPB}$ (related to the placement and release of aortic cross-clamps during cardiac surgery) but not in ECMO. ${ }^{9,10}$

In this study, we used a porcine neonatal model of ECMO to investigate the hypothesis that rising plasma concentrations of inflammatory cytokines during ECMO reflect de novo synthesis of these mediators in inflamed tissues, and therefore, can be used to assess the severity of ECMO-related SIRS. We subjected previously healthy piglets weighing $3-6 \mathrm{~kg}$ to venoarterial ECMO in the laboratory and detected inflammatory changes that were similar to those seen in human neonates. However, plasma concentrations of tumor necrosis factor-alpha (TNF- $\alpha$ ) and interleukin-8 (IL-8, CXCligand 8 (CXCL8)) increased within the first $2 \mathrm{~h}$ of ECMO, faster than the increase in tissue expression of these cytokines. These findings indicated that the increased plasma concentrations of inflammatory cytokines could not be completely explained on the basis of increased de novo synthesis in inflamed tissues and may have resulted, at least partially, from redistribution of preformed stores of these cytokines. Plasma TNF- $\alpha$ and IL-8 levels correlated with plasma mast cell tryptase activity, indicating that mast cell degranulation may contribute to the rapid rise of plasma cytokine concentrations during ECMO.

\section{METHODS}

\section{Neonatal Porcine ECMO}

Animals

Mixed-breed neonatal piglets of either gender weighing 3-6 kg were subjected to venoarterial ECMO after approval by the UAB Institutional Animal Care and Use Committee. Sham animals received anesthesia, assisted ventilation, vascular cannulation, and heparinization similar to ECMO animals, but were not connected to the circulatory pump device. Data in this study represent eight animals each in sham and ECMO groups; three animals in each group were euthanized after $2 \mathrm{~h}$ of treatment and the remaining five after $8 \mathrm{~h}$.

\section{Anesthesia, assisted ventilation and baseline support}

The piglets were anesthetized with intramuscular atropine $(0.04 \mathrm{mg} / \mathrm{kg})$, tiletamine $\mathrm{HCl} /$ zolazepam $\mathrm{HCl}(4.4 \mathrm{mg} / \mathrm{kg})$, and xylazine $(4.4 \mathrm{mg} / \mathrm{kg})$. All animals were intubated with a size 3.5 cuffed endotracheal tube and mechanically ventilated (volume controlled, tidal volume $=15 \mathrm{ml} / \mathrm{kg}$, Hallowell EMC 2000 veterinary anesthesia ventilator) at a rate of $10-15$ cycles/min with the pig in a dorsally recumbent position. Anesthesia was maintained by inhalation of isoflurane (1.5-2.5\%) administered in $100 \%$ oxygen. Surface lead II EKG recordings, arterial blood pressure (ABP), and core (rectal) temperature was monitored. Water-heated pads were used to maintain normothermia $\left(37 \pm 1^{\circ} \mathrm{C}\right)$. Serum electrolyte levels and arterial blood gases were monitored throughout the study. Animals were given intravenous $0.9 \%$ saline at $2-5 \mathrm{ml} / \mathrm{kg} / \mathrm{h}$ during the experiment and also provided boluses as required to maintain normal $\mathrm{ABP}$.

\section{Vascular access}

A multiple side-hole $8-10 \mathrm{~F}$ Biomedicus venous cannula (Medtronic Perfusion Systems, Minneapolis, MN, USA) was inserted into the external jugular vein and advanced into the right atrium. The common carotid artery was cannulated using a 6-8 F Biomedicus arterial cannula. Placement of these cannulae was confirmed by fluoroscopy.

\section{Heparinization}

After circuit priming and before starting ECMO, animals were bolused with porcine heparin (Sigma, St Louis, MO, USA; $150 \mathrm{U} / \mathrm{kg}$ ), followed by additional intravenous heparin injections to maintain activated clotting times of $180-220 \mathrm{~s}$ after the initiation of ECMO.

\section{ECMO procedure}

Extracorporeal membrane oxygenation circuit was primed with $110.9 \%$ saline followed by $300 \mathrm{ml}$ porcine whole blood collected from a similarly anesthetized pig, $10 \mathrm{ml}$ sodium bicarbonate, $1500 \mathrm{U}$ of porcine heparin, and $50 \mathrm{mg}$ calcium chloride. The venoarterial ECMO system consisted of a Biomedicus BP-50 centrifugal pump (Medtronic, Shoreview, MN, USA), and a Minimax hollow fiber oxygenator (Medtronic) with an integral heat exchanger. Water bath temperatures were maintained at $37^{\circ} \mathrm{C}$ using a Sarns Dual Heater/Cooler (Soma Technology, Cheshire, CT, USA). Gas flow rates to the membrane oxygenator were maintained at $0.5 \mathrm{l} / \mathrm{min}$ of $100 \%$ oxygen. After starting ECMO, the flow 
rates in the circuit were advanced to $350 \mathrm{ml} / \mathrm{min}$ or $1.5 \mathrm{l} / \mathrm{min} / \mathrm{m}^{2}$. Additional volume requirements to maintain circuit flow or desired hemoglobin level were met by the addition of a porcine whole blood or saline boluses. Animals were euthanized after 2 or $8 \mathrm{~h}$ of ECMO by administration of potassium chloride.

\section{Neutrophils}

Neutrophils were isolated using an established densitycentrifugation method. ${ }^{11}$ Briefly, porcine blood was mixed with $4 \%$ gelatin (Sigma) in $0.9 \% \mathrm{NaCl}$ and allowed to settle at $37^{\circ} \mathrm{C}$ for $30 \mathrm{~min}$. The supernatant was then layered over Histopaque-1083 separation media (Sigma) and centrifuged at $400 \mathrm{~g}$ for $25 \mathrm{~min}$ at room temperature. Neutrophils were isolated from the resultant pellet after hypotonic lysis of the red blood cells and suspended in Hanks' balanced salt solution (Sigma). ${ }^{12}$ Cells were suspended in RPMI-1640 supplemented with $10 \%$ fetal bovine serum and antibiotics.

\section{Histopathological Analysis and Immunohistochemistry}

Harvested tissues were fixed in formalin and embedded in paraffin. Histopathological examinations were performed on hematoxylin-eosin stained sections. Paraffin-embedded tissue sections were immunostained for c-kit (CD117, a mast cell marker), TNF- $\alpha$, and IL-8/CXCL8 as previously described. ${ }^{12,13}$ Briefly, tissue sections were deparaffinized with xylene and graded ethanols and antigen retrieval was achieved by heating the sections in $10 \mathrm{mM}$ sodium citrate buffer, $\mathrm{pH} 6.0$ at $95^{\circ} \mathrm{C} \times 10 \mathrm{~min}$ followed by treatment with proteinase $\mathrm{K}(20 \mu \mathrm{g} / \mathrm{ml}$; Sigma $)$ at $37^{\circ} \mathrm{C} \times 20 \mathrm{~min}$. After blocking overnight (Superblock, Pierce, Rockford, IL, USA), the sections were stained overnight $\left(4^{\circ} \mathrm{C}\right)$ with primary antibodies (mouse antibodies against c-kit and goat antibodies against TNF- $\alpha$, and IL- 8 from Santa Cruz Biotechnology, Santa Cruz, CA, USA). Secondary staining was performed at room temperature for $30 \mathrm{~min}$ with Alexa 488- and/or Alexa 568-conjugated donkey anti-goat IgG and rabbit anti-mouse IgG (Invitrogen, San Diego, CA, USA). Controls included slides with no primary antibody and appropriate isotype control. The cell nuclei were stained with 4',6-diamidino-2phenylindole (Calbiochem, San Diego, CA, USA), diluted 1:1000 in PBS, applied for 3 min. Imaging was performed using a fluorescence microscope (Carl Zeiss Microimaging, Thornwood, NY, USA).

\section{Flow Cytometry}

Neutrophils were stained for neutrophil activation markers CD18, CD35, CD62L, and CD11b using our previously reported protocol. ${ }^{14}$ Controls included cells with no antibody and appropriate isotype control.

\section{Real-Time PCR}

Real-time PCR was carried out using our previously described protocol. ${ }^{14}$ Total RNA was isolated using the acid guanidinium thiocyanate-phenol-chloroform extraction protocol (Trizol reagent, Invitrogen). First-strand cDNA was synthesized using oligo-dT primers and Moloney murine leukemia virus reverse transcriptase (Invitrogen). Real-time PCR primers were designed using the Beacon Design software (Bio-Rad, Hercules, CA, USA). Two-step real-time PCR was carried out using a SYBR Green protocol described elsewhere. ${ }^{14}$ Data were normalized against glyceraldehyde-3phosphate dehydrogenase and gene expression was compared between samples by using the $2^{-\Delta \Delta \mathrm{CT}}$ method.

\section{ELISA}

Plasma concentrations of porcine cytokines TNF- $\alpha$, IL-8/ CXCL8, IL-6, and IL-1 $\beta$ were measured by enzyme-linked immunosorbent assay (ELISA) (R\&D systems, Minneapolis, MA, USA). ELISA kits for porcine CRP and C5a were purchased from Alpco Diagnostics, Salem, NH, USA, and Immuno-biological Laboratories, Minneapolis, MN, USA, respectively.

\section{Western Blots}

Western blots were performed for TNF- $\alpha$ using our previously described protocol ${ }^{14}$ and were analyzed using the NIH scion software.

\section{Measurement of Mast Cell Degranulation}

We used a commercially available assay (Millipore, Bedford, MA, USA) based on the spectophotometric detection $(405 \mathrm{~nm})$ of a chromophore $p$-nitroaniline (pNA) released by tryptase action on a labeled substrate tosyl-gly-pro-lys-pNA.

\section{Archived Tissues from Human Neonates Treated with ECMO}

Autopsy samples from full-term human neonates who died during treatment with ECMO for pulmonary hypertension $(n=3)$ were obtained with approval of the local Institutional Review Board.

\section{Statistical Methods}

Parametric and nonparametric tests were applied using the SigmaStat 3.1.1 software (Systat, Point Richmond, CA, USA). The number of samples and the statistical analyses are described in the figure legends. A $P$-value of $<0.05$ was considered significant.

\section{RESULTS}

\section{Hemodynamic Changes During ECMO}

The ECMO animals developed tachycardia and hypotension within 1-2 $\mathrm{h}$ of initiation of ECMO. We treated hypotension with intravenous boluses of normal saline or porcine wholeblood transfusions (given if hematocrit was $<20 \%$ ). In the ECMO group, capillary leakiness became clinically evident by about $4 \mathrm{~h}$ of ECMO with abdominal distension due to ascites. Animals subjected to ECMO needed more frequent fluid boluses and blood transfusions (total administered volume $18 \pm 9 \mathrm{ml} / \mathrm{kg}$ in sham animals $v s 178 \pm 16 \mathrm{ml} / \mathrm{kg}$ in animals 
treated with ECMO, $P<0.05)$. The vital parameters of animals in sham and ECMO groups did not differ from each other.

\section{Histopathological Evidence of Inflammation}

Leukocyte infiltration, focal hemorrhages, and/or edema were evident in most tissues examined, including the lung, intestine, liver, skin, and kidney after $2 \mathrm{~h}$ of ECMO. These inflammatory changes increased in severity after $8 \mathrm{~h}$. These changes were most prominent in the lung and the intestine (Figure 1a). These histopathological changes of inflammation in our porcine model were generally similar to changes noted in autopsy tissue samples from human neonates who died during ECMO (Figure 1b). Although the contribution of the underlying illness (that led to cardiopulmonary failure) to the inflammatory changes in these neonates cannot be clearly ascertained, these findings provide indirect evidence for our observations in the porcine model.

\section{Neutrophil Activation During ECMO}

As neutrophil activation is a key event during human ECMO,${ }^{15,16}$ we measured the expression of CD18, CD35, CD62L, and CD11b on circulating neutrophils in hourly samples from sham and ECMO animals by flow cytometry. Compared with sham animals, the expression of all four activation markers was significantly upregulated in ECMO animals. The expression of these markers at $2 \mathrm{~h}$ (time point chosen to compare with histopathological changes of inflammation) is depicted in Figure 1c.

\section{Porcine ECMO was Associated with a 'Global' Activation of Proinflammatory Genes}

Using a real-time PCR microarray, we next measured mRNA expression of 48 well-characterized porcine inflammatory genes in lung and intestinal tissue. As shown in Figure 2a, we detected increased expression of neutrophil chemokines IL-8/ CXCL8 and growth-related oncoprotein- $\beta /$ CXCL2 and mononuclear cell chemokines interferon-inducible protein10/CXCL10, CXCL12, macrophage inflammatory protein-1 $\beta /$ CC-ligand 4, CCL11, and CCL21. Inflammatory cytokines, such as TNF- $\alpha$, interferon- $\gamma$, IL-1 $\beta$, IL-6, IL-12, IL-15, and IL-18 were also upregulated. We also found increased tissue expression of the cognate receptors for TNF- $\alpha$ (TNF-receptor II), IL8 and GRO- $\beta /$ CXCL2 (CXC receptors CXCR1 and CXCR2), CXCL10 (CXCR3), and MIP-1//CCL4 (CCR3). The expression of nuclear factor $-\kappa \mathrm{B}$ and activator protein 1 transcription factors was also increased. These changes showed that ECMO activated a wide range of proinflammatory genes encoding for important chemokines, cytokines, cytokine/chemokine receptors, and critical transcriptional factors that regulate the expression of these cytokines and chemokines.

\section{Increased Plasma Concentrations of Proinflammatory Cytokines}

In support of the data obtained from the PCR array shown in Figure $4 \mathrm{a}$, we compared the plasma concentrations of TNF- $\alpha$, IL-8, IL-6, and IL-1 $\beta$ in sham and ECMO animals. We detected a rapid rise in plasma levels of TNF- $\alpha$ and IL-8 within the first $2 \mathrm{~h}$ of ECMO, which was not observed in sham-treated animals (Figure 2b). Plasma IL-1 $\beta$ and IL-6 levels increased more slowly, rising significantly after $4-6 \mathrm{~h}$ of ECMO.

\section{Onset of Systemic Inflammatory Response during ECMO was not Reflected in Leukocyte Counts or Plasma CRP Concentrations}

We also investigated whether the onset of the systemic inflammatory response after initiation of ECMO was associated with changes in blood leukocyte counts or plasma CRP concentrations, two acute-phase reactants that are frequently used in the clinical setting. Total leukocyte counts were $13.1 \pm 2 \times 10^{9} / 1$ and $12.4 \pm 3.2 \times 10^{9} / 1$ at $4 \mathrm{~h}$ of treatment in sham $v$ E ECMO animals, respectively (differences not statistically significant). The absolute neutrophil counts were also not affected by ECMO $\left(8.9 \pm 1.2 \times 10^{9} / 1\right.$ vs $7.85 \pm 2.1 \times 10^{9} / 1$ in sham and ECMO animals, respectively). Similarly, plasma CRP concentrations were also not altered by ECMO-related SIRS (Figure 3). Other acute-phase reactants, such as erythrocyte sedimentation rate and fibrinogen levels, were considered but not evaluated during ECMO because of the presence of multiple confounding factors such as transfusions and heparinization.

\section{Rapid rise in plasma TNF- $\alpha$ and IL-8 levels during ECMO is not matched by increased synthesis of these cytokines in the tissues}

To investigate whether increased plasma concentrations of TNF- $\alpha$ and IL-8 during ECMO reflect increased de novo synthesis of these cytokines in inflamed tissues, we next measured mRNA and protein expression for TNF- $\alpha$ and IL- 8 in tissues harvested from sham and ECMO animals after $2 \mathrm{~h}$ of treatment. Although the mRNA expression of TNF- $\alpha$ increased in various tissues at $2 \mathrm{~h}$ of ECMO (Figure $4 \mathrm{a}$ ), we did not detect important differences in TNF- $\alpha$ protein that could explain the rise in plasma TNF- $\alpha$ concentrations during ECMO at this time point. In contrast to the eightfold increase in plasma TNF- $\alpha$ concentration at $2 \mathrm{~h}$ of ECMO, TNF- $\alpha$ expression was decreased in the intestine and increased 1.5- to 2-fold in tissue samples from the liver and spleen (Figure 4b). Similarly, we measured a 0.9- to 5-fold change in IL- 8 mRNA in the tissues but the amount of IL-8 protein did not increase significantly in the tissues after $2 \mathrm{~h}$ of ECMO; the expression of IL-8 was also decreased in the intestine at the 2-h time point (Supplementary Figure 1). 
a
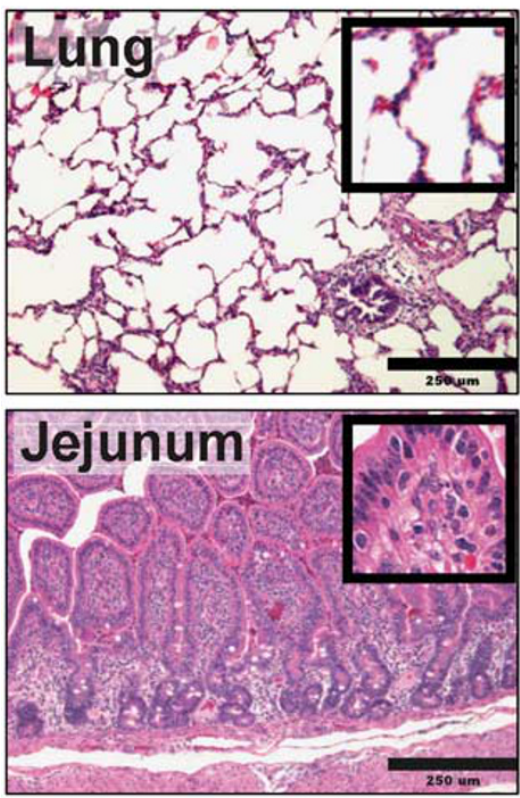

SHAM 8 hrs
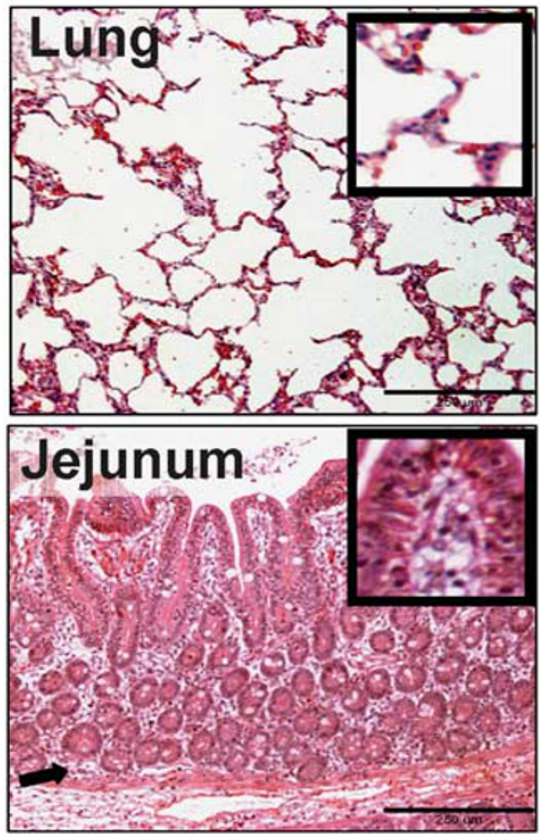

ECMO 2 hrs
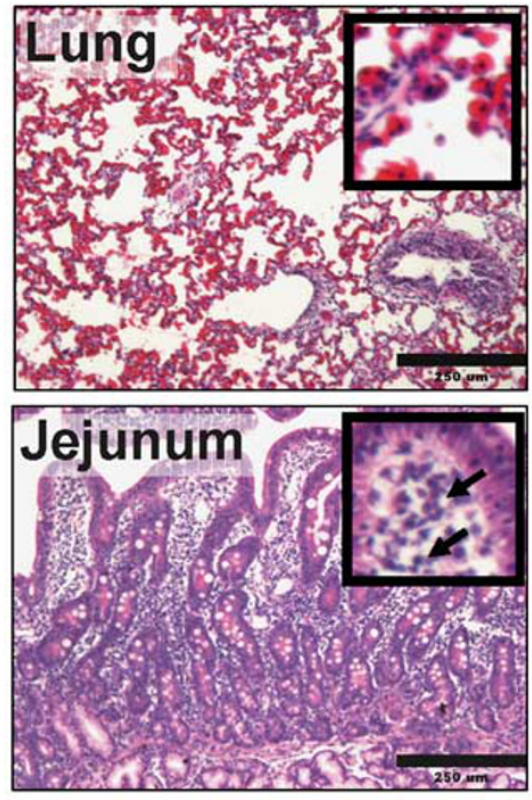

ECMO 8 hrs
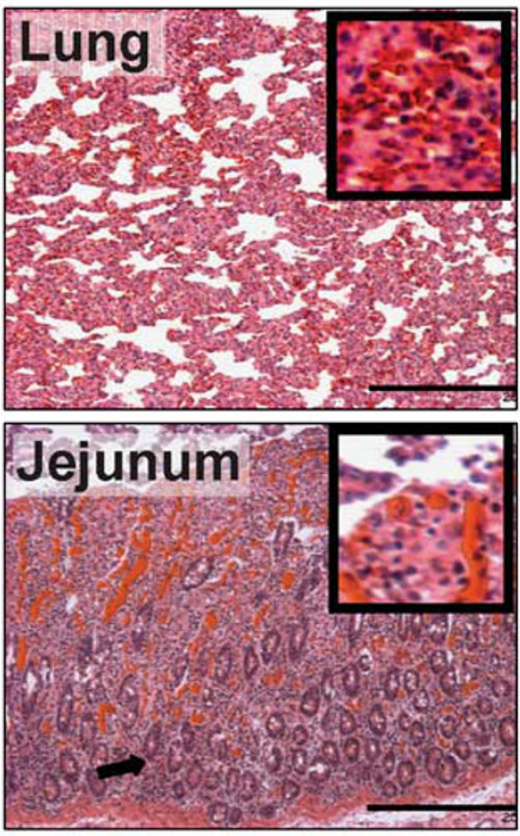

b
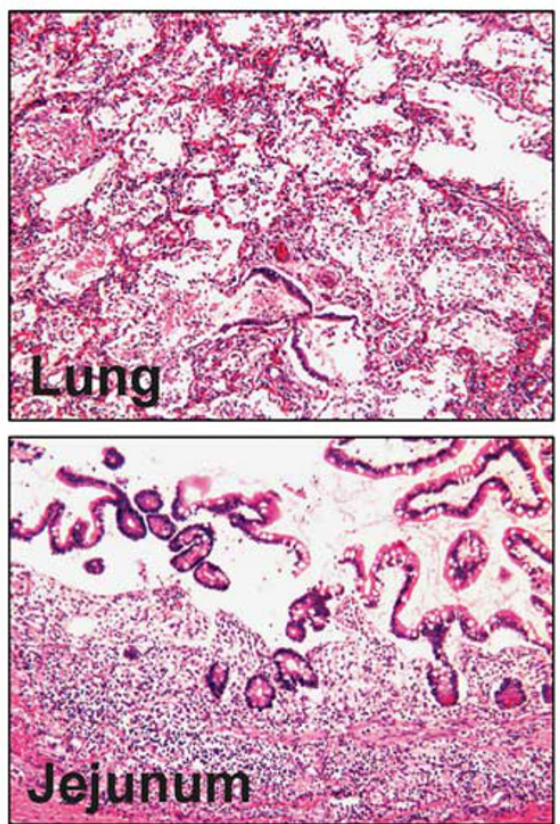

Figure 1 Histopathological changes of inflammation during extracorporeal membrane oxygenation (ECMO). (a) Hematoxylin-eosin (H\&E)-stained sections from the lung and the intestine (jejunum) after 2 and $8 \mathrm{~h}$ of ECMO. Upper panel: Histopathological changes after $2 \mathrm{~h}$ of ECMO. Photomicrographs (magnification $\times 100$ ) highlight the differences between the near-normal alveolar histoarchitecture in sham animals vs the conspicuous leukocyte infiltration and focal hemorrhages in ECMO. In the intestine, ECMO caused an increase in cellularity in the lamina propria (low magnification), which was due to leukocyte infiltration (higher magnification inset). Data represents $n=3$ animals in both groups. Lower panel: Histopathological changes after $8 \mathrm{~h}$ of ECMO. Inflammatory changes in the lung became worse with increased leukocyte infiltration, hemorrhages, and septal edema. In the intestine, there was an increase in leukocyte infiltration (black arrows) and focal hemorrhages. The epithelium was disrupted (magnification $\times 100$ ). Insets show high-magnification photomicrographs $(\times 400)$ highlighting the inflammatory changes. Data represent $n=5$ animals in both groups. (b) Photomicrographs of the lung and jejunum from human neonates who died during ECMO, showing the marked similarity between inflammatory changes in our porcine model and human tissues. Upper panel shows the effect of ECMO on the lung, including leukocyte infiltration and alveolar hemorrhages. Lower panel shows marked leukocyte infiltration and disruption of the epithelium in the intestine. Data represent three neonates. (c) Neutrophil activation during neonatal porcine ECMO. Representative FACS histograms from sham and ECMO animals drawn after $2 \mathrm{~h}$ of treatment show increased expression of activation markers CD18, CD35, $\mathrm{CD} 62 \mathrm{~L}$, and CD11b on circulating neutrophils during ECMO. Bar diagrams shown below the FACS panels summarize the information from an $n=5$ in both groups. Data were analyzed by the Mann-Whitney $U$-test. Asterisk $\left(^{*}\right)$ indicates a significant difference between ECMO and sham groups, $P<0.05$. 

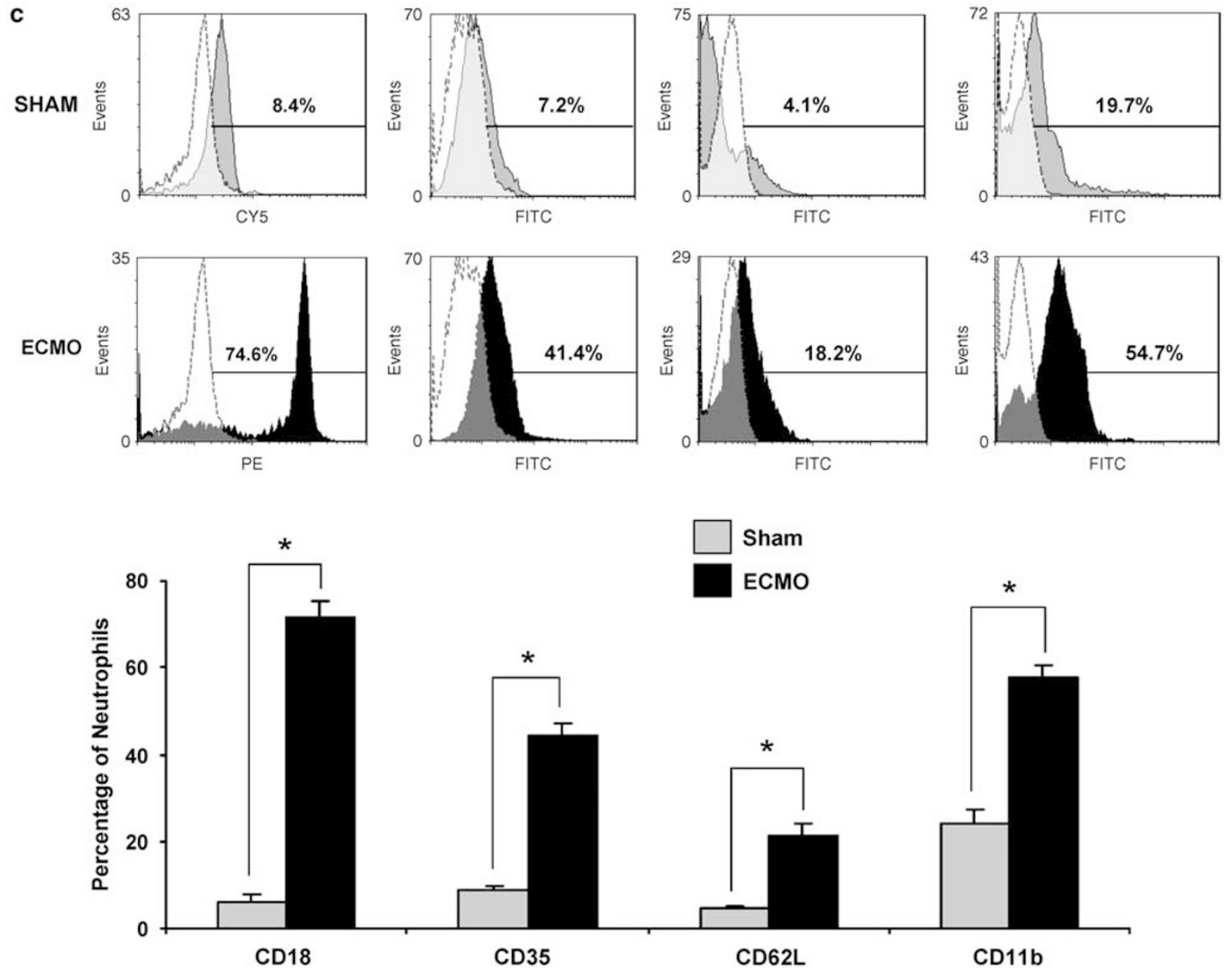

Sham

ECMO

CD35

Figure 1 continued.

\section{Mast Cells Contain Preformed TNF- $\alpha$}

As the rapid rise in plasma TNF- $\alpha$ and IL-8 levels during ECMO could not be explained on the basis of increased de novo synthesis of these cytokines, we next investigated whether ECMO mobilized preformed tissue stores of TNF- $\alpha$ and IL- 8 into the plasma. As seen in the western blots in Figure $4 \mathrm{~b}$, the intestine contained the largest amounts of TNF- $\alpha$ in control animals. These data were consistent with evidence that mast cells present in mucosal organs such as the intestine comprise the largest store of preformed TNF- $\alpha$ in the body ${ }^{17,18}$ and have an important role in experimental models of SIRS. ${ }^{19}$ In Figure $4 \mathrm{~b}$, the reduction in TNF- $\alpha$ in the ECMO intestine was also consistent with the hypothesized release of cellular stores during ECMO. Therefore, we first used immunofluorescence microscopy to confirm whether porcine mucosal mast cells contained TNF- $\alpha$. As seen in Figure $5 \mathrm{a}$, strong immunoreactivity for TNF- $\alpha$ was detected in c-kit ${ }^{+}$mast cells in the intestine in both sham and ECMO animals. We also detected TNF- $\alpha$ in mast cells in sham/ECMO porcine lung, although the lung contained fewer mast cells than the intestine (not depicted). Mast cells also showed strong immunoreactivity for IL-8 in both sham and ECMO animals (not depicted). To confirm the pathophysiological relevance of mast cells in ECMO-related SIRS in human neonates, we also stained intestinal tissues from human neonates treated with ECMO (autopsy samples); a similar colocalization of c-kit and TNF- $\alpha$ was detected (Figure 5b).

\section{ECMO is Associated with Degranulation of Mast Cells}

To investigate whether the initiation of ECMO in our porcine model was associated with mast cell degranulation, we next compared plasma samples from sham and ECMO animals for tryptase (mast cell protease 7) activity, a serine protease present almost exclusively and in large amounts in mast cell granules. ${ }^{20,21}$ As shown in Figure $5 c$, plasma tryptase activity after $1 \mathrm{~h}$ of ECMO was nearly fourfold higher than in sham animals and correlated with plasma concentrations of TNF- $\alpha$ and IL-8/CXCL8 $(P<0.05)$. Mast cell degranulation 
a EXPRESSION OF PRO-INFLAMMATORY GENES

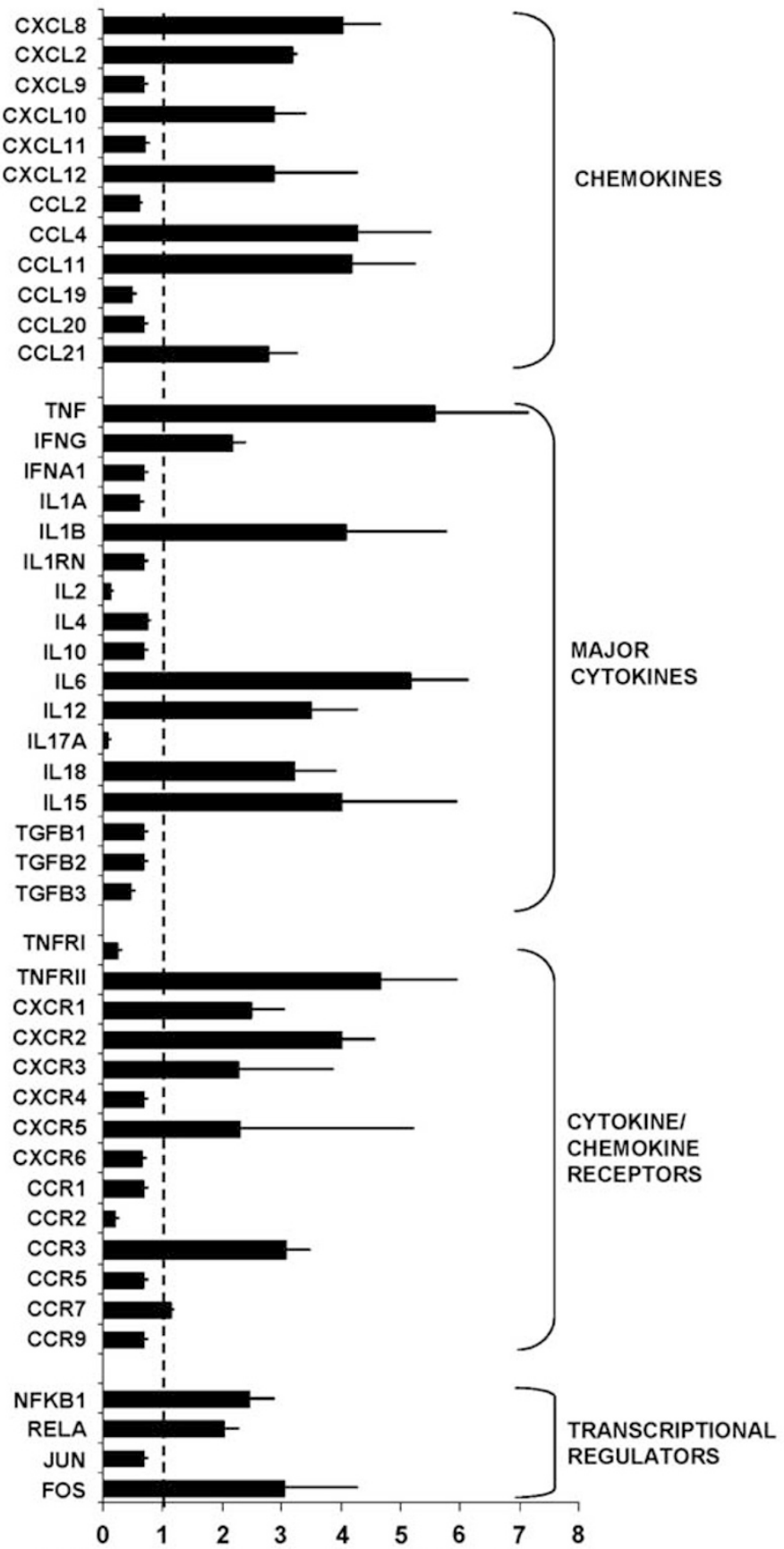

Fold change in gene expression above sham b PLASMA CYTOKINES
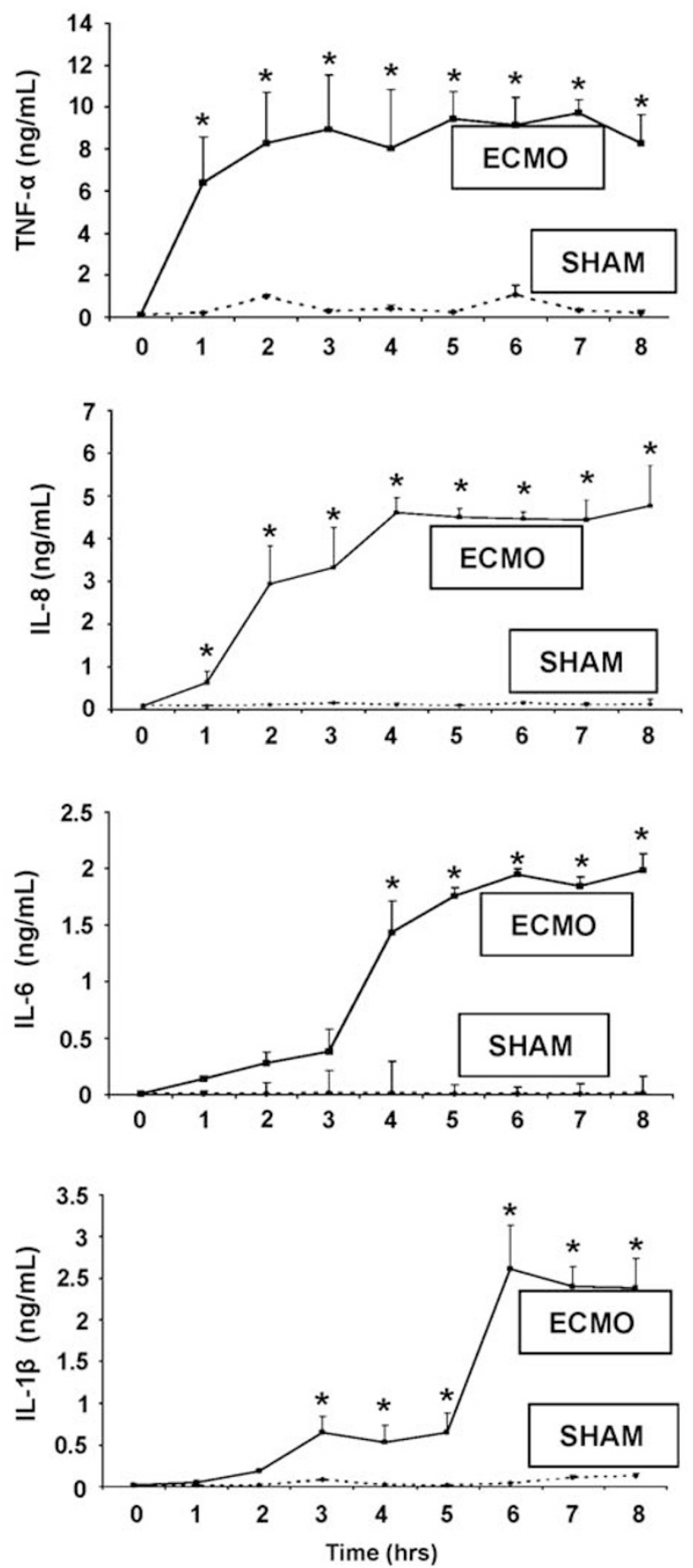

Figure 2 'Global' activation of inflammatory mediators in lung tissue during porcine neonatal extracorporeal membrane oxygenation (ECMO). (a) Real-time PCR microarray profiles of mRNA expression of various proinflammatory genes in the lung after $8 \mathrm{~h}$ of ECMO emphasize the ' $\mathrm{global}$ ' activation of inflammatory mediators during ECMO. Data represent an $n=5$ animals in both sham and ECMO groups and are depicted as mean \pm s.e.m. fold change above sham (dashed line). Gene expression profiles in the intestine were generally similar to those in the lung (not depicted). (b) Increased plasma concentrations of proinflammatory cytokines tumor necrosis factor-alpha (TNF- $\alpha$ ), interleukin (IL)-8/CXC-ligand 8 (CXCL8), IL-6, and IL- $1 \beta$ as measured by enzyme-linked immunosorbent assay. Line diagrams depict cytokine concentrations $(n=5$ animals in sham and ECMO group; means \pm s.e.m.). Asterisk $\left({ }^{*}\right)$ indicates a significant difference between ECMO and sham groups, $P<0.05$. Data were analyzed by the repeated measures ANOVA on ranks. 


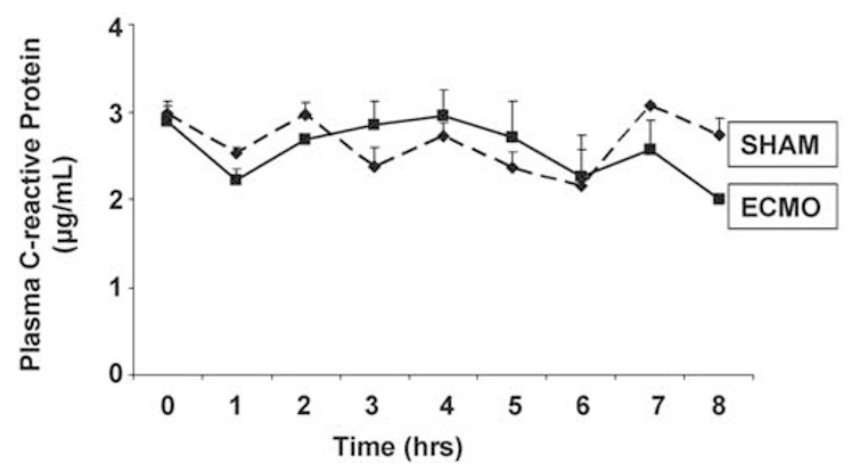

Figure 3 Onset of systemic inflammatory response during extracorporeal membrane oxygenation (ECMO) was not reflected in plasma C-reactive protein concentrations during ECMO. Unlike the marked changes seen in plasma cytokine concentrations, we did not detect significant changes in plasma CRP in the initial $8 \mathrm{~h}$ of ECMO. Other acute-phase reactants such as leukocyte counts were also not discriminatory (not depicted). Line diagrams (means \pm s.e.m.) summarize information from five animals each in ECMO and sham groups. Data were analyzed by the repeated measures ANOVA on ranks.

is a plausible event during ECMO because activation of the complement pathway in the membrane oxygenator releases several mast cell secretagogues, such as C5a, C3a, and C3adesArg. ${ }^{22}$ Consistent with this hypothesis, we detected increased plasma C5a levels in our porcine model after $1 \mathrm{~h}$ of ECMO (inset, Figure 5c).

\section{DISCUSSION}

The histopathological changes of inflammation, activation of neutrophils, and increased plasma concentrations of various proinflammatory cytokines in our piglet model were generally similar to those reported in human ECMO. ${ }^{16,23-25}$ Existing clinical and experimental evidence on the mechanisms of ECMO-related SIRS emphasizes the activation of neutrophils and other leukocytes as a pivotal event during ECMO, which occurs due to contact with the foreign surface of the circuit, shear stress, expression of inflammatory cytokines, activation of complement, coagulation and fibrinolytic pathways, and increased concentrations of bioactive lipids. ${ }^{16,22-28}$ These activated neutrophils adhere to the capillary/venular endothelium and undergo degranulation to produce cytokines, arachidonic acid metabolites, and reactive oxygen species, causing widespread microvascular injury ${ }^{15,27}$ and multiorgan dysfunction. ${ }^{16,22-28}$

The rapid rise in plasma concentrations of TNF- $\alpha$ and IL- 8 almost immediately after the initiation of ECMO suggests that these cytokines may be important mediators in the development of ECMO-related inflammation. TNF- $\alpha$ can induce the expression of a wide range of inflammatory genes, including its own, ${ }^{29}$ which suggests that the early rise in circulating TNF- $\alpha$ levels during ECMO may form a 'feedforward' loop to augment the inflammatory responses.

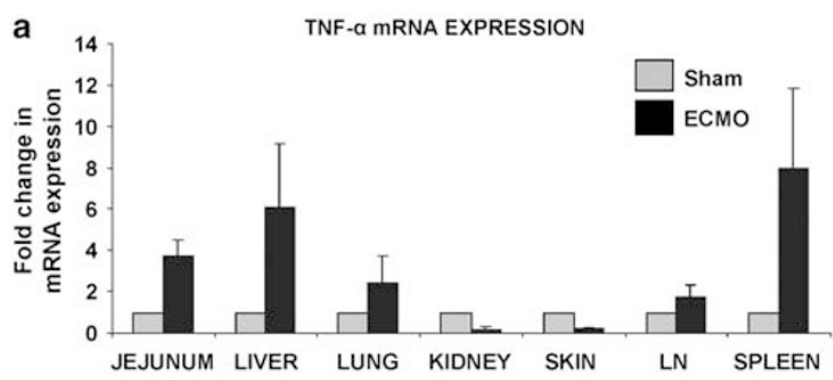

b

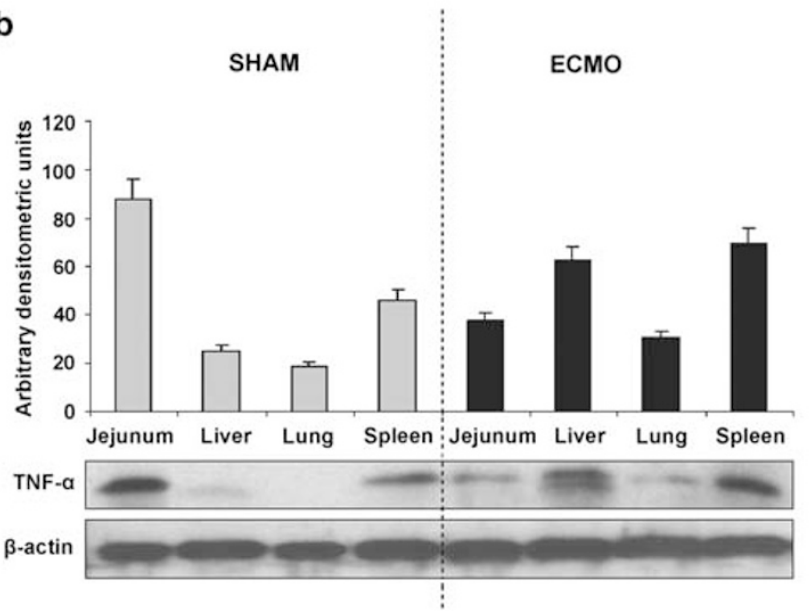

Figure 4 Rapid rise in plasma tumor necrosis factor-alpha (TNF- $\alpha$ ) concentrations during extracorporeal membrane oxygenation (ECMO) is not matched by increased synthesis of TNF- $\alpha$ protein in the tissues. We harvested the intestine, liver, lung, kidney, skin, mesenteric lymph nodes, and the spleen after $2 \mathrm{~h}$ of ECMO. Although the mRNA expression for TNF- $\alpha$ was increased as anticipated, we did not detect an increase in TNF- $\alpha$ protein proportionate to the increase in plasma TNF- $\alpha$ concentrations. (a) Bar diagrams show the fold change (means \pm s.e.m.) in TNF- $\alpha$ mRNA as measured by real-time PCR after $2 \mathrm{~h}$ of treatment. Data represent an $n=3$ animals in sham and ECMO groups. (b) Western blots for porcine TNF- $\alpha$ and $\beta$-actin on tissue samples from the intestine, liver, lung, and spleen (the four tissues with the greatest increase in expression of TNF- $\alpha$ mRNA above). Bar diagrams show the densitometric analysis (means \pm s.e.m.) of these bands. Data are representative of three animals in each group.

In human neonates, higher plasma TNF- $\alpha$ concentrations have been noted in patients who died during ECMO than in 'responders' who recovered completely. ${ }^{16,26,30}$ In other studies, plasma concentrations of IL-8 correlated with the severity of SIRS and cardiopulmonary impairment during ECMO. ${ }^{16,23,24,31}$

The marked elevation in plasma cytokine levels in our model contrasted with the low sensitivity of acute-phase reactants, such as leukocyte counts and plasma CRP concentrations. Elevated CRP levels have been noted in one previous report on human $\mathrm{ECMO}^{31}$ in which CRP concentrations were higher in nonresponders as compared with patients who improved on ECMO, although CRP was found to be less sensitive as a prognostic indicator than IL-6. The differential expression of IL-6 and CRP in our model was 
a

SHAM

ECMO

b

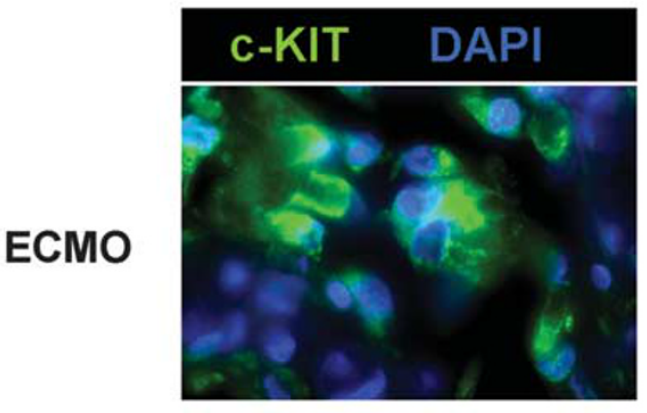

JEJUNUM FROM HUMAN NEONATES TREATED WITH ECMO
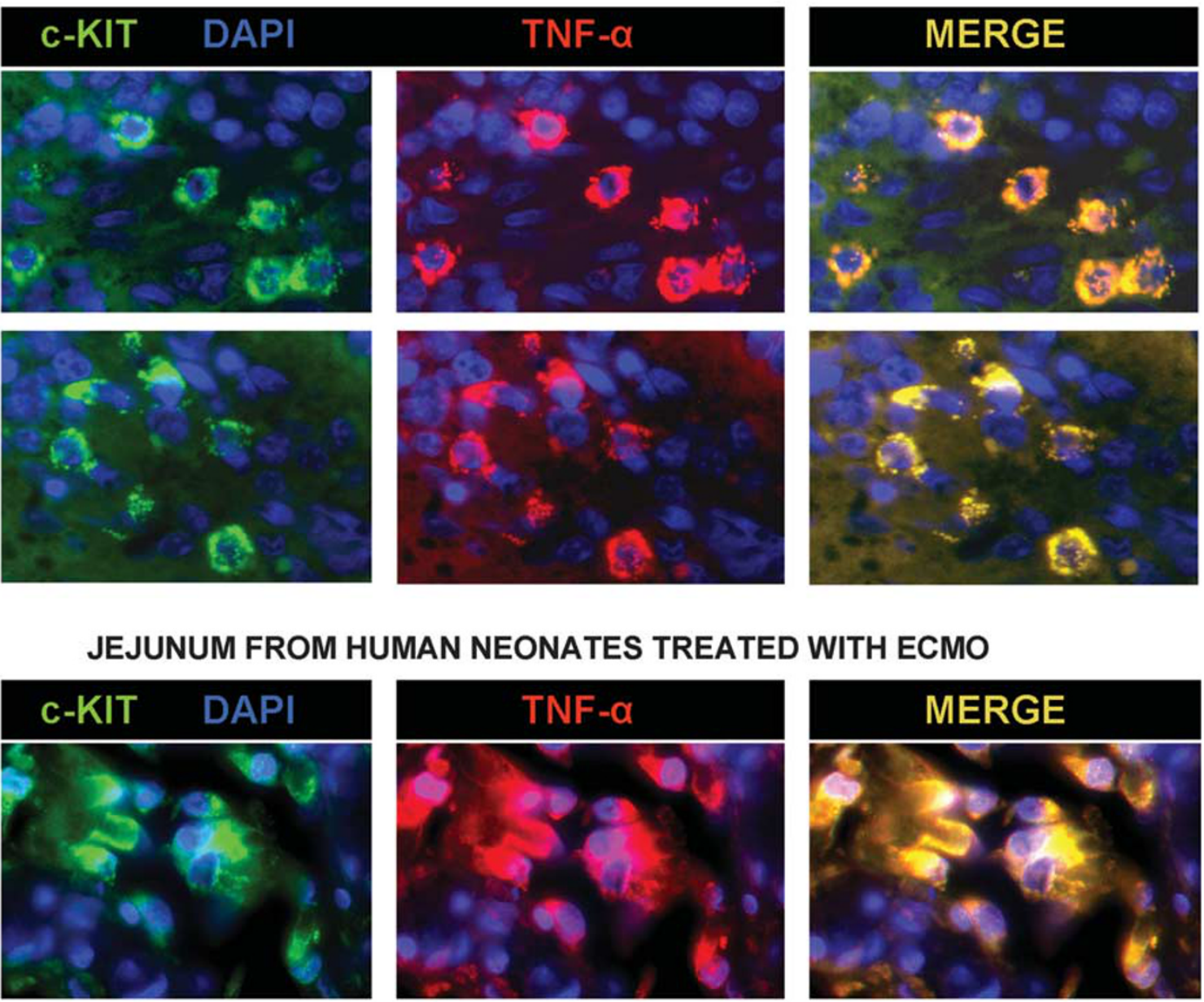

PLASMA C5

CONCENTRATIONS

C PLASMA TRYPTASE ACTIVITY
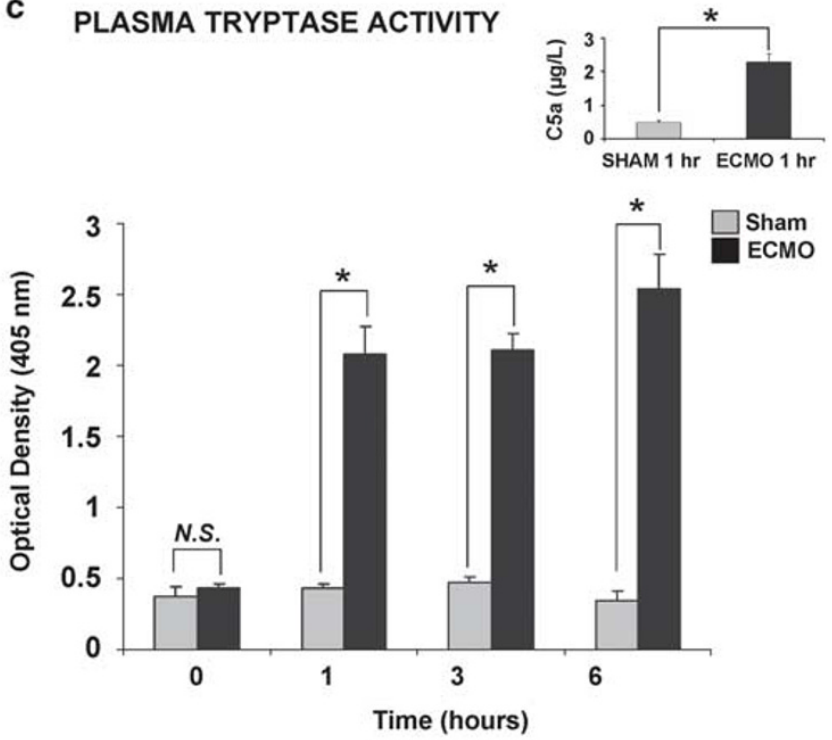
surprising because IL-6 is a potent inducer of CRP production in the liver and the two mediators are usually identified as bound variables in various inflammatory states. ${ }^{32} \mathrm{~A}$ possible explanation may be related to the 8 -h duration of our experiments, in which we might have missed a late rise in plasma CRP concentrations (following the delayed rise in IL-6 levels). Alternatively, the CRP response may have been dampened by the relative resistance of porcine hepatocytes to IL-6, a species-specific feature. IL-6 induces CRP expression in hepatocytes through a CCAAT-enhancer binding proteinbeta $(\mathrm{C} / \mathrm{EBP}-\beta)$-mediated pathway, ${ }^{33}$ which carries unique post-translational modifications and/or compartmentalization of C/EBP- $\beta$ in pigs. ${ }^{34}$

The increase in plasma TNF- $\alpha$ and IL-8 concentrations within $2 \mathrm{~h}$ of initiation of ECMO in our model was not associated with a proportionate increase in the tissue synthesis of these cytokines at the same time. Considering the rapid change in plasma cytokine levels and also because the expression of TNF- $\alpha$ protein was actually decreased in the intestine at $2 \mathrm{~h}$, we hypothesized that ECMO mobilized these cytokines from preformed tissue stores of TNF- $\alpha$ into plasma. Of all the major cellular sources of TNF- $\alpha$, mast cells $^{35}$ and eosinophils ${ }^{36}$ are the only cell types considered capable of storing preformed TNF- $\alpha$. We focused on mast cells and not eosinophils in this study because the gut mucosa normally contains a large reservoir of mast cells. ${ }^{17,18}$ The plausibility of mast cell degranulation, as shown in our model, is supported by existing data on the activation of complement and consequent release of mast cell secretagogues, such as C5a, C3a, and C3adesArg during human ECMO ${ }^{37} \mathrm{C} 3 \mathrm{a}$ levels have also been shown to correlate with plasma TNF- $\alpha$ levels and the severity of inflammation during ECMO. $^{30}$

Mast cells are now increasingly recognized as a major source of preformed TNF- $\alpha$ in SIRS in diverse settings, such as in Gram-negative and Gram-positive bacterial sepsis, peritonitis, severe alveolar hypoxia, and portal hypertension. ${ }^{38-42}$ Unlike other major cellular sources of TNF- $\alpha$ such as macrophages and T-/B-lymphocytes that contain little or no detectable TNF- $\alpha$ unless appropriately stimulated, ${ }^{43,44}$ mast cells present in mucosal organs contain large amounts of preformed TNF- $\alpha^{17,18}$ and have an important role in experimental models of SIRS. ${ }^{19}$ Histamine liberation has been previously reported during $\mathrm{CPB}$ in older children and adults, but circulating basophils, and not mast cells, were incriminated as the source. ${ }^{45,46}$ As we mentioned earlier, mechanistic differences in the inflammatory responses associated with ECMO vs $\mathrm{CPB}$ are not unexpected and illustrate the need for specifically directed studies for these two forms of ECC. Even though ECMO and CPB are both performed using similar circulatory pumps and other major equipment, $\mathrm{CPB}$ is associated with several additional pathophysiological variables such as ischemia-reperfusion injury from aortic cross-clamping and due to the vasoconstrictive effects of hypothermia and hemodilution, proinflammatory effects of surgical trauma and tissue debris, and more frequent use of protamine. ${ }^{7,8,47-49}$

In conclusion, the institution of ECMO in our porcine model induced systemic inflammatory changes that were remarkably similar to those reported during human ECMO. Although our original hypothesis that increased plasma TNF- $\alpha /$ IL-8 levels reflect tissue synthesis of these cytokines during ECMO was disproven, our data reaffirmed the higher sensitivity of inflammatory cytokines as indicators of inflammation during ECMO as compared with blood leukocyte counts and C-reactive protein. Further studies are needed to develop a panel of cytokines that are synthesized de novo during ECMO-related SIRS and not released from preformed stores. We have also identified mast cell degranulation and the release of TNF- $\alpha$ as early events in ECMO-related SIRS. The availability of agents that can stabilize enteric mast cells $^{50}$, as well as effective neutralizing anti-TNF- $\alpha$ antibodies raises important therapeutic possibilities ${ }^{51}$ and indicates a need to aggressively investigate these targets in preclinical and clinical settings.

Supplementary Information accompanies the paper on the Laboratory Investigation website (http://www.laboratoryinvestigation.org)

\section{ACKNOWLEDGEMENTS}

This study was supported in part by the American Heart Association Grant 0665155B, American Gastroenterological Association 2006 Research Scholar Award, and the NIH awards K12HD043397, RHD059142 (to AM). The work was made possible in part by the Research Facilities Improvement Grant C06RR15490 from the National Center for Research Resources.

Figure 5 (a) Mast cells in the sham/extracorporeal membrane oxygenation (ECMO) porcine intestine contain preformed tumor necrosis factor-alpha $\left(\right.$ TNF- $\alpha$ ). Immunofluorescence photomicrographs $(\times 1000)$ from the intestine show strong TNF- $\alpha$ immunoreactivity in c-kit/CD117 ${ }^{+}$mast cells in both sham animals and after $2 \mathrm{~h}$ of ECMO. TNF- $\alpha$ immunoreactivity was slightly weaker in ECMO animals than in the sham group, consistent with our findings of mast cell degranulation during ECMO. Data are representative of three to five stained sections from different animals in both sham and ECMO groups. (b) Similar colocalization of c-kit and TNF- $\alpha$ seen in archived autopsy tissues from human neonates who died during ECMO. Data represent three different neonates. (c) Porcine neonatal ECMO was associated with degranulation of mast cells. Bar diagrams (means \pm s.e.m.) show plasma tryptase activity in sham and ECMO animals as a function of time. Plasma tryptase activity was significantly increased after $1 \mathrm{~h}$ of ECMO, indicating that ECMO was associated with mast cell degranulation. The data summarize information from an $n=5$ animals in both sham and ECMO groups. Statistical comparisons were made by repeated measures ANOVA on ranks. Asterisk $\left(^{*}\right)$ indicates a significant difference between ECMO and sham groups, $P<0.05$. Inset: Bar diagram (means \pm s.e.m.) shows that plasma samples after $1 \mathrm{~h}$ of ECMO contained high levels of $C 5 \mathrm{a}$, a potent mast cell secretagogue released during activation of the complement pathway. Data were analyzed by the Mann-Whitney U-test. Asterisk $\left.{ }^{*}\right)$ indicates a significant difference between ECMO and sham groups, $P<0.05$. 


\section{DISCLOSURE/CONFLICT OF INTEREST}

The authors declare no conflict of interest.

1. Kelly Jr RE, Phillips JD, Foglia RP, et al. Pulmonary edema and fluid mobilization as determinants of the duration of ECMO support. J Pediatr Surg 1991;26:1016-1022.

2. Ford JW. Neonatal ECMO: current controversies and trends. Neonatal Netw 2006;25:229-238.

3. Khoshbin $\mathrm{E}$, Dux $A \mathrm{E}$, Killer $\mathrm{H}$, et al. A comparison of radiographic signs of pulmonary inflammation during ECMO between silicon and poly-methyl pentene oxygenators. Perfusion 2007;22:15-21.

4. Butler J, Pathi VL, Paton RD, et al. Acute-phase responses to cardiopulmonary bypass in children weighing less than 10 kilograms. Ann Thorac Surg 1996;62:538-542.

5. Kozik DJ, Tweddell JS. Characterizing the inflammatory response to cardiopulmonary bypass in children. Ann Thorac Surg 2006;81: S2347-S2354.

6. Zahraa JN, Moler FW, Annich GM, et al. Venovenous versus venoarterial extracorporeal life support for pediatric respiratory failure: are there differences in survival and acute complications? Crit Care Med 2000;28:521-525.

7. Ganapathy S, Murkin JM, Dobkowski W, et al. Stress and inflammatory response after beating heart surgery versus conventional bypass surgery: the role of thoracic epidural anesthesia. Heart Surg Forum 2001;4:323-327.

8. Brix-Christensen V. The systemic inflammatory response after cardiac surgery with cardiopulmonary bypass in children. Acta Anaesthesiol Scand 2001;45:671-679.

9. Walker LK, Short BL, Traystman RJ. Impairment of cerebral autoregulation during venovenous extracorporeal membrane oxygenation in the newborn lamb. Crit Care Med 1996;24:2001-2006.

10. Kuratani T, Matsuda $\mathrm{H}$, Sawa $\mathrm{Y}$, et al. Experimental study in a rabbit model of ischemia-reperfusion lung injury during cardiopulmonary bypass. J Thorac Cardiovasc Surg 1992;103:564-568.

11. Stahl GL, Morse DS, Martin SL. Eicosanoid production from porcine neutrophils and platelets: differential production with various agonists Am J Physiol 1997;272(6 Pt 1):C1821-C1828.

12. Maheshwari A, Kurundkar AR, Shaik SS, et al. Epithelial cells in fetal intestine produce chemerin to recruit macrophages. Am J Physiol Gastrointest Liver Physiol 2009;297:G1-G10.

13. Smythies LE, Maheshwari A, Clements R, et al. Mucosal IL-8 and TGF-beta recruit blood monocytes: evidence for cross-talk between the lamina propria stroma and myeloid cells. J Leukoc Biol 2006:80:492-499.

14. Shaik SS, Soltau TD, Chaturvedi G, et al. Low-intensity shear stress increases endothelial ELR+ CXC chemokine production via a FAKP38beta MAPK-NF-kappa B pathway. J Biol Chem 2009;284:5945-5955

15. DePuydt LE, Schuit KE, Smith SD. Effect of extracorporeal membrane oxygenation on neutrophil function in neonates. Crit Care Med 1993;21:1324-1327.

16. Fortenberry JD, Bhardwaj V, Niemer $\mathrm{P}$, et al. Neutrophil and cytokine activation with neonatal extracorporeal membrane oxygenation. J Pediatr 1996;128(5 Pt 1):670-678.

17. Gordon JR, Galli SJ. Mast cells as a source of both preformed and immunologically inducible TNF-alpha/cachectin. Nature 1990;346: 274-276.

18. Gordon JR, Galli SJ. Release of both preformed and newly synthesized tumor necrosis factor alpha (TNF-alpha)/cachectin by mouse mast cells stimulated via the Fc epsilon RI. A mechanism for the sustained action of mast cell-derived TNF-alpha during lgE-dependent biological responses. J Exp Med 1991;174:103-107.

19. Tang C, Lan C, Wang C, et al. Amelioration of the development of multiple organ dysfunction syndrome by somatostatin via suppression of intestinal mucosal mast cells. Shock 2005;23: 470-475.

20. He $\mathrm{S}, \mathrm{Xie} \mathrm{H}$. Modulation of tryptase and histamine release from human lung mast cells by protease inhibitors. Asian Pac J Allergy Immunol 2004;22:205-212.

21. Shalit M, Schwartz LB, Golzar N, et al. Release of histamine and tryptase in vivo after prolonged cutaneous challenge with allergen in humans. J Immunol 1988;141:821-826.
22. Graulich J, Sonntag J, Marcinkowski M, et al. Complement activation by in vivo neonatal and in vitro extracorporeal membrane oxygenation. Mediators Inflamm 2002;11:69-73.

23. Underwood MJ, Pearson JA, Waggoner J, et al. Changes in 'inflammatory' mediators and total body water during extra-corporeal membrane oxygenation (ECMO). A preliminary study. Int J Artif Organs 1995;18:627-632.

24. Kawahito K, Misawa Y, Fuse K. Extracorporeal membrane oxygenation support and cytokines. Ann Thorac Surg 1998;65: 1192-1193.

25. Adrian K, Mellgren K, Skogby M, et al. Cytokine release during long-term extracorporeal circulation in an experimental model. Artif Organs 1998;22:859-863.

26. Mildner RJ, Taub N, Vyas JR, et al. Cytokine imbalance in infants receiving extracorporeal membrane oxygenation for respiratory failure. Biol Neonate 2005;88:321-327.

27. Graulich J, Walzog B, Marcinkowski M, et al. Leukocyte and endothelial activation in a laboratory model of extracorporeal membrane oxygenation (ECMO). Pediatr Res 2000;48: 679-684.

28. Graves 3rd ED, Loe WA, Redmond CR, et al. Extracorporeal membrane oxygenation as treatment of severe meconium aspiration syndrome. South Med J 1989;82:696-698.

29. Dempsey PW, Doyle SE, He JQ, et al. The signaling adaptors and pathways activated by TNF superfamily. Cytokine Growth Factor Rev 2003;14:193-209.

30. Plotz FB, van Oeveren $\mathrm{W}$, Bartlett $\mathrm{RH}$, et al. Blood activation during neonatal extracorporeal life support. J Thorac Cardiovasc Surg 1993;105:823-832.

31. Risnes I, Wagner K, Ueland T, et al. Interleukin- 6 may predict survival in extracorporeal membrane oxygenation treatment. Perfusion 2008;23:173-178.

32. Herzum I, Renz H. Inflammatory markers in SIRS, sepsis and septic shock. Curr Med Chem 2008;15:581-587.

33. Cha-Molstad H, Young DP, Kushner I, et al. The interaction of C-Rel with C/EBPbeta enhances C/EBPbeta binding to the C-reactive protein gene promoter. Mol Immunol 2007;44:2933-2942.

34. Schrem H, Kleine M, Borlak J, et al. Physiological incompatibilities of porcine hepatocytes for clinical liver support. Liver Transpl 2006;12:1832-1840.

35. Gurish MF, Boyce JA. Mast cells: ontogeny, homing, and recruitment of a unique innate effector cell. J Allergy Clin Immunol 2006;117: 1285-1291.

36. Spencer LA, Szela CT, Perez SA, et al. Human eosinophils constitutively express multiple Th1, Th2, and immunoregulatory cytokines that are secreted rapidly and differentially. J Leukoc Biol 2009;85: 117-123.

37. Hocker JR, Wellhausen SR, Ward RA, et al. Effect of extracorporeal membrane oxygenation on leukocyte function in neonates. Artif Organs 1991;15:23-28.

38. Mallen-St Clair J, Pham CT, Villalta SA, et al. Mast cell dipeptidyl peptidase I mediates survival from sepsis. J Clin Invest 2004;113: 628-634.

39. Sutherland RE, Olsen JS, McKinstry A, et al. Mast cell IL-6 improves survival from Klebsiella pneumonia and sepsis by enhancing neutrophil killing. J Immunol 2008;181:5598-5605.

40. Nautiyal KM, McKellar H, Silverman AJ, et al. Mast cells are necessary for the hypothermic response to LPS-induced sepsis. Am J Physiol Regul Integr Comp Physiol 2009;296:R595-R602.

41. Chao J, Wood JG, Gonzalez NC. Alveolar hypoxia, alveolar macrophages, and systemic inflammation. Respir Res 2009;10:54.

42. Tang CW, Lan C, Liu R. Increased activity of the intestinal mucosal mast cells in rats with multiple organ failure. Chin J Dig Dis 2004;5: 81-86.

43. Old LJ. Tumor necrosis factor (TNF). Science 1985;230:630-632.

44. Cuturi MC, Murphy M, Costa-Giomi MP, et al. Independent regulation of tumor necrosis factor and lymphotoxin production by human peripheral blood lymphocytes. J Exp Med 1987;165:1581-1594.

45. Seghaye MC, Duchateau J, Grabitz RG, et al. Histamine liberation related to cardiopulmonary bypass in children: possible relation to transient postoperative arrhythmias. J Thorac Cardiovasc Surg 1996;111:971-981 
46. Withington $\mathrm{DE}$, Aranda JV. Histamine release during cardiopulmonary bypass in neonates and infants. Can J Anaesth 1997;44:610-616.

47. Yeh $\mathrm{CH}$, Chen TP, Lee $\mathrm{CH}$, et al. Cardioplegia-induced cardiac arrest under cardiopulmonary bypass decreased nitric oxide production which induced cardiomyocytic apoptosis via nuclear factor kappa B activation. Shock 2007;27:422-428.

48. Asimakopoulos G. Systemic inflammation and cardiac surgery: an update. Perfusion 2001;16:353-360.
49. Ko WJ, Chen YS, Lee YC. Replacing cardiopulmonary bypass with extracorporeal membrane oxygenation in lung transplantation operations. Artif Organs 2001;25:607-612.

50. Penissi AB, Rudolph MI, Villar M, et al. Effect of dehydroleucodine on histamine and serotonin release from mast cells in the isolated mouse jejunum. Inflamm Res 2003;52:199-205.

51. Choo-Kang BS, Hutchison S, Nickdel MB, et al. TNF-blocking therapies: an alternative mode of action? Trends Immunol 2005;26:518-522. 